\title{
Asymmetry in the discrimination of quantity by rats: The role of the intertrial interval
}

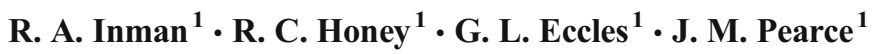

Published online: 24 July 2015

(C) The Author(s) 2015. This article is published with open access at Springerlink.com

\begin{abstract}
In three experiments, rats were trained to discriminate between 20 and five (Exps. 1 and 2), or between 40 and five (Exp. 3), black squares. The squares were randomly distributed in the center of a white background and displayed on a computer screen. For one group, the patterns containing the higher quantity of squares signaled the delivery of sucrose $(+)$, whilst patterns with the lower quantity of squares did not (-). For the second group, sucrose was signaled by the lower, but not by the higher, quantity of squares. In Experiment 1, the intertrial interval (ITI) was a white screen, and the $20+/ 5-$ discrimination was acquired more readily than the $5+/ 20$ discrimination. For Experiment 2, the ITI was made up of 80 black squares on a white background. In this instance, the $5+/ 20$ - discrimination was acquired more successfully than the 20+/5-discrimination. In Experiment 3, two groups were trained with a $40+/ 5-$ discrimination, and two with a $5+/ 40$ discrimination. For one group from each of these pairs, the training trials were separated by a white ITI, and the $40+/ 5-$ discrimination was acquired more readily than the 5+/40discrimination. For the remaining two groups, the training trials were not separated by an ITI, and the two groups acquired the task at approximately the same rate. The results indicate that the cues present during the ITI play a role in the asymmetrical acquisition of magnitude discriminations based on quantity.
\end{abstract}

Keywords Discrimination learning $\cdot$ Stimulus generalization · Quantity discrimination

\section{R. A. Inman}

inmanr@cardiff.ac.uk

1 School of Psychology, Cardiff University, Cardiff, Wales CF10 3AT, UK
A small and diverse body of evidence has shown an asymmetry in the acquisition of discriminations based on stimulus magnitude: When two stimuli of different magnitudes signal the presence and absence of a reinforcer, the discrimination between them develops more rapidly when the signal for the reinforcer, $\mathrm{S}+$, is larger rather than smaller than the signal for the absence of the reinforcer, S-. This effect has been found with discriminations based on different intensities of white noise, using conditioned suppression with rats (Zieliński \& Jakubowska, 1977); different intensities of odors, using appetitive conditioning with bees (Pelz, Gerber, \& Menzel, 1997); different lengths of panels, using escape from a swimming pool with rats (Kosaki, Jones, \& Pearce, 2013); different durations of an auditory stimulus, using appetitive conditioning with rats (Kyd, Pearce, Haselgrove, Amin, \& Aggleton, 2008; Todd, Winterbauer, \& Bouton, 2010); and different numbers of the same objects, for appetitive conditioning with brown bears (Vonk \& Beran, 2012) and pigeons (Inman, Honey, \& Pearce, 2015; Watanabe, 1998).

To explain this asymmetry, Kosaki et al. (2013) pointed to the influence of the similarity between $\mathrm{S}+$ and the background cues, especially those present during the intertrial interval (ITI). The rationale behind this analysis is considered in some detail in the General Discussion. For the present, it is sufficient to note that Kosaki et al. proposed that the easier it is to discriminate between the occasions when $\mathrm{S}+$ is present and absent, the more readily will excitatory responding develop to $\mathrm{S}+$, and thus the more readily will the discrimination between $\mathrm{S}+$ and S- develop. Clearly, it will normally be easier to discriminate between $\mathrm{S}+$ and the background cues when the former is of high rather than low magnitude, which will then lead to an asymmetry in the acquisition of discriminations based on stimulus magnitude.

Although the just mentioned results are consistent with the proposals of Kosaki et al. (2013), they do not confirm that the 
asymmetry observed with magnitude discriminations depends on the influence of the cues present during the ITI. As a step toward providing such evidence, Inman et al. (2015) conducted a series of autoshaping experiments with pigeons using the stimuli depicted in Fig. 1, which were presented on a television screen behind a response key. The screen was white during the intertrial interval, and either five or 20 black squares were displayed for the training trials. The discrimination was easier to solve when food was signaled by 20 but not by five squares, $20+/ 5-$, than with the opposite arrangement, $5+/ 20$. In order to test whether the outcome of this magnitude discrimination was indeed influenced by the similarity between $\mathrm{S}+$ and the ITI stimulation, Inman et al. conducted a further experiment with the training stimuli displayed in Fig. 1, but with 288 squares displayed on the screen throughout the ITI. The purpose of this manipulation was to ensure that the stimulation during the ITI was more similar to the large-magnitude than to the small-magnitude training stimulus. This modification should now result in the cues present during the ITI being more similar to the patterns with 20 squares than to those with five squares, and result in the 20+/5- discrimination being more difficult than the 5+/20- discrimination. The results confirmed this prediction.

The initial purpose of the present experiments was to determine whether the results obtained with pigeons by Inman et al. (2015) could also be found with rats. Thus far, using rats, an asymmetry has been found between discriminations based on the intensity of sound (Zieliński \& Jakubowska, 1977), the length of an object (Kosaki et al., 2013), and the duration of an auditory cue (Kyd et al., 2008). It remains to be determined with this species whether a similar asymmetry can be found when the discrimination involves differences in quantity. Experiment 1 was based on the design depicted in Fig. 1, and revealed a similar pattern of results to that reported by Inman et al., using appetitive Pavlovian conditioning. The remaining two experiments were then conducted to assess the role played by the cues present during the ITI on the asymmetry revealed in Experiment 1. In Experiment 2, the cues present during the ITI were made more similar to the large-quantity, rather than the small-quantity, conditioned stimulus (CS), in order to determine whether this manipulation would reverse the asymmetry. For Experiment 3 we adopted a novel design to assess the effect of conducting the quantity discrimination without an ITI. If the stimulation during the ITI played a crucial role in the asymmetry observed with the discriminations in Experiment 1, then conducting training without an ITI would abolish the asymmetry.

\section{Experiment 1}

Two groups of rats received appetitive Pavlovian conditioning in chambers from which they could view the stimuli depicted in Fig. 1 on the screen of a computer monitor. Sucrose solution could be delivered to a dispenser on the outside of the wall nearest the monitor, and access to this dispenser was made possible by a small hole in the wall. Sucrose solution was available after patterns with 20 squares, but not after five squares, for the $20+/ 5$ group, whereas the opposite arrangement was used for the $5+/ 20$ group. As training progressed, it was expected that the frequency with which a rat's snout was inserted into the hole above the sucrose dispenser would be greater during patterns that signaled the imminent delivery of sucrose, rather than no delivery of sucrose. If this pattern of results should emerge, it would indicate for the first time that this species is capable of solving magnitude discriminations based on different numbers of the same visual object. More importantly, on the basis of the proposals of

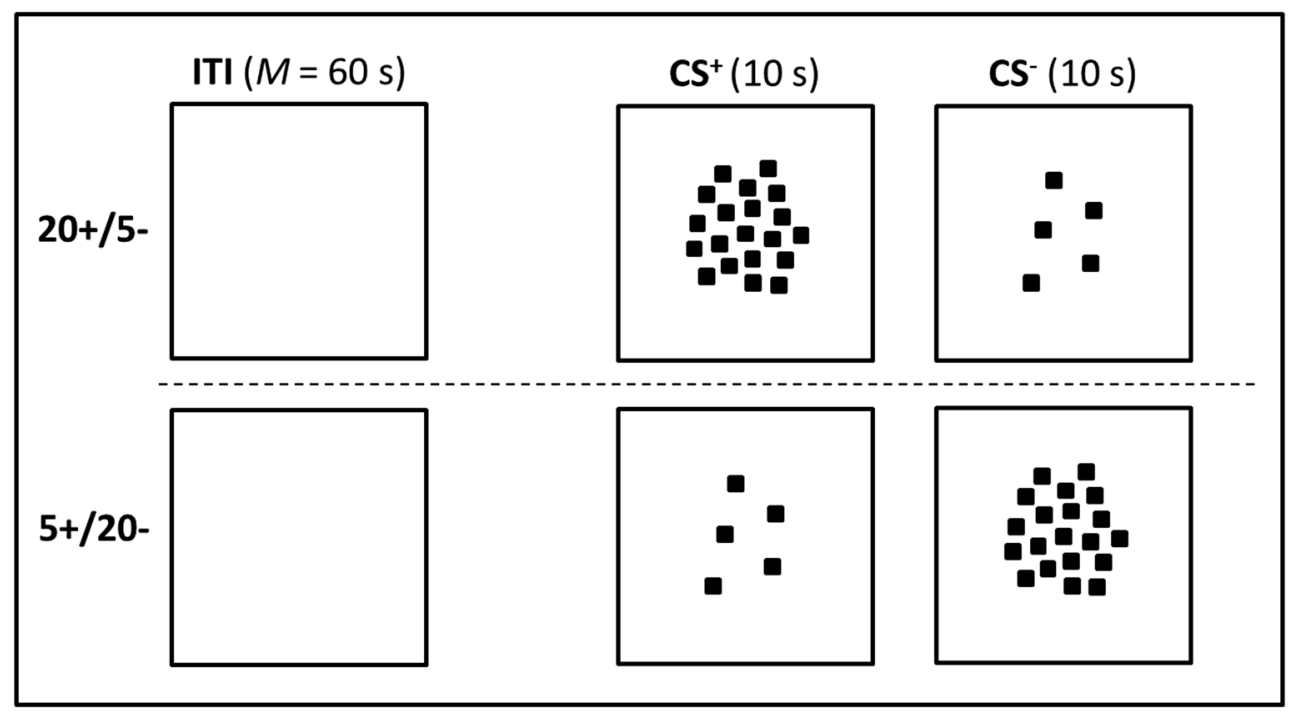

Fig. 1 The stimuli used in Inman, Honey, and Pearce (2015) and in Experiment 1. The figure is presented for illustrative purposes and does not depict accurately the images used in the experiments. 
Kosaki et al. (2013), the discrimination was expected to be acquired more readily by the $20+/ 5-$ group than the $5+/ 20$ - group.

\section{Method}

Subjects The subjects were 32 male hooded Lister rats supplied by Harlan Olac (Bicester, Oxon, UK). Their mean freefeeding weight was $303 \mathrm{~g}$. They were housed in pairs in a temperature-controlled colony room (approximately $20^{\circ} \mathrm{C}$ ) that was continuously illuminated for $12 \mathrm{~h}$ per day, with lights on at 07:00. They had access to water ad libitum but were food-deprived to between $80 \%$ and $85 \%$ of their freefeeding weights prior to the start of the experiment. They were maintained at this weight by being fed a restricted diet after each experimental session. The rats had previously been used for an appetitive conditioning experiment for which they were divided into two groups that received different auditory discriminations. Prior to the present experiment, they were randomly assigned to the two new groups $(n=16)$, with the constraint that each of the new groups contained eight rats from each of the former groups.

Apparatus Eight operant chambers were used. The side walls $(28 \times 30 \mathrm{~cm}, \mathrm{H} \times \mathrm{W})$ and ceiling of each chamber were constructed from clear Perspex. The floor was a metal grid positioned $5 \mathrm{~cm}$ above the base of the chamber that was lined with an absorbent, odor-removing paper. In the center of the back wall was a circular hole, $3 \mathrm{~cm}$ in diameter, the center of which was $3 \mathrm{~cm}$ above the grid floor. The circular hole allowed access to a well into which sucrose solution ( $8 \%$ sugar, 92\% water) was delivered. This area will henceforth be referred to as the magazine. The number of snout entries made into the magazine was recorded using an infrared sensor. Beyond the magazine, at a distance of $8 \mathrm{~cm}$, a $34 \times 27 \mathrm{~cm}$ computer monitor was placed to present the visual stimuli. The lower edge of the screen was in line with the chamber floor; a peristaltic pump located beneath each conditioning chamber delivered the sucrose solution via a plastic tube into the well. A PC with Whisker software, and programmed in Visual Basic 6.0, controlled the experimental events and recorded the number of snout entries. Throughout the experimental sessions, the testing room lights were switched off so that the only illumination came from the stimuli presented on the computer monitors. Barriers were placed between the chambers to prevent the animals from seeing each other.

Stimuli The training stimuli consisted of either five or 20 filled black squares $(1 \times 1 \mathrm{~cm})$ that were randomly arranged within a circular area $(20 \mathrm{~cm}$ in diameter) in the center of a white background. Ten different arrangements of both stimuli were used. The squares in the five- and 20-square stimuli were separated by mean distances of $4.5 \mathrm{~cm}(S D=2.4 \mathrm{~cm})$ and
$1.8 \mathrm{~cm}(S D=0.6 \mathrm{~cm})$, respectively. During the ITI the computer monitors were illuminated white.

Procedure Rats were first given two sessions in which they were trained to approach the magazine in order to obtain sucrose. These hour-long sessions consisted of the regular delivery of $1 \mathrm{ml}$ of sucrose, once a minute, every minute, for 30 min, and then a further $30 \mathrm{~min}$ in which no sucrose was delivered. During these sessions the computer monitors were switched off. The rats then received 14 60-min sessions of discrimination training. Within each session, nine trials had patterns showing five squares, and nine trials had patterns showing 20 squares. The order of the trials was determined randomly with the restriction that a trial type could not be repeated more than twice consecutively. Each stimulus was presented for $15 \mathrm{~s}$ at a time, and successive trials were separated by an ITI of 2, 3, or $4 \mathrm{~min}(M=3 \mathrm{~min})$. The duration of each ITI was determined randomly, but with the constraint that each of the three possible intervals was selected six times in each session. During the ITI the computer monitors were illuminated entirely white. For the $20+/ 5$ - group, the presentation of 20 squares was immediately followed by the delivery of $1 \mathrm{ml}$ of sucrose solution, and the presentation of five squares was not. For the $5+/ 20-$ group, five squares signaled the delivery of sucrose, and 20 squares signaled the absence of sucrose.

Data analysis The individual mean rates of responding, based on the numbers of times the beam of the infrared sensor was interrupted during a trial, were recorded for all trials in each of the 14 sessions of discrimination training. The rates of responding during the ITI before every trial were also recorded. For the sake of clarity, and to simplify the analysis, the raw data for each session were transformed into discrimination ratios. The ratios were of the form $A /(A+B)$, where $A$ and $B$ were the mean rates of responding on reinforced $(\mathrm{CS}+)$ and nonreinforced (CS-) trials, respectively. A ratio greater than .50 indicated that more nose pokes were made during CS+ than CS-. The analysis of these ratios was conducted with analyses of variance (ANOVAs) using a rejection criterion of $p<.05$. The reported effect size for an ANOVA with more than one factor is partial eta squared $\left(\eta_{\mathrm{p}}{ }^{2}\right)$, whereas for comparisons between two means it is eta squared $\left(\eta^{2}\right)$. For both measures of effect size, $95 \%$ confidence intervals (CIs) were computed using the method reported by Steiger (2004).

\section{Results and discussion}

The mean rates of responding to $\mathrm{CS}+$ and $\mathrm{CS}-$ and throughout every ITI for each of the 14 experimental sessions are presented in Fig. 2. The rats in both groups eventually responded more frequently during $\mathrm{CS}+$ than $\mathrm{CS}-$, but the magnitude of 
this discrimination was more pronounced in the $20+/ 5-$ than in the $5+/ 20$ - group. Figure 2 also presents the results as discrimination ratios, which, not surprisingly, show that the discrimination was acquired more readily by the $20+/ 5-$ than by the $5+/ 20-$ group.

Analysis of the individual mean discrimination ratios with an ANOVA confirmed that the observed difference between the two groups was significant, $F(1,30)=49.80, p<.001, \eta_{\mathrm{p}}{ }^{2}$ $=.62,95 \%$ CI $[.37, .75]$. The analysis also revealed a significant main effect of session, $F(13,390)=6.46, p<.001, \eta_{\mathrm{p}}{ }^{2}=$ $.18,95 \%$ CI $[.09, .22]$, but the Session $\times$ Group interaction, $F$ $<1$, was not significant.

Inspection of the mean rates of ITI responding, which are displayed in the left-hand and center panels of Fig. 2, shows that the rate of responding was generally more rapid in the $5+/ 20$ group than in the $20+/ 5-$ group. A two-way ANOVA of the individual mean rates of responding during the ITIs for each of the 14 sessions revealed significant effects of group, $F(1,30)=$ $4.99, p=.033, \eta_{\mathrm{p}}{ }^{2}=.14,95 \%$ CI $[.00, .36]$, and session, $F(13$, $390)=5.17, p<.000, \eta_{\mathrm{p}}{ }^{2}=.15,95 \%$ CI $[.06, .18]$. The Group $\times$ Session interaction was not significant, $F<1$.

The results demonstrated, for the first time with rats, an asymmetry in a magnitude discrimination based on different quantities of the same visual object. The present findings therefore extend the generality of the results reported by Inman et al. (2015), who conducted a similar experiment using autoshaping with pigeons. The results also support the proposal of Kosaki et al. (2013) that the acquisition of magnitude discriminations is influenced by the similarity between $\mathrm{S}+$ and the cues present during the ITI. When the similarity between $\mathrm{S}+$ and the ITI cues is close, as was the case for the $5+20$ - discrimination, the discrimination will be harder to solve than when this difference is more pronounced, as was the case for the 20+5-discrimination.

\section{Experiment 2}

The two groups of rats in Experiment 2 received the same training as the two groups in Experiment 1, except that instead of being entirely white during the ITI, the television screen displayed 80 black squares clustered around the center. The purpose of this manipulation was to render the stimulation that was present during the ITI more similar to the patterns containing 20 black squares than those containing five black squares. According to the proposals of Kosaki et al. (2013), this manipulation should result in a reversal of the asymmetry seen in Experiment 1, with the 20+/5- discrimination being harder to solve than the $5+/ 20-$ discrimination. This prediction has already been confirmed with pigeons by Inman et al. (2015). In their experiment, 288 black squares were displayed against a white screen during the ITI, and the patterns used for the training trials contained either five or 20 squares.

Of course, it is possible that the proposals of Kosaki et al. (2013) do not apply to rats, and that the asymmetry in Experiment 1 may have occurred for quite a different reason than the one they put forward. For example, the salience of 20 squares might be regarded as being greater than that of five squares. Theories of conditioning (e.g., Rescorla \& Wagner, 1972) would then predict that excitatory conditioning would progress more rapidly with 20 squares than with five squares, and, irrespective of the stimulation present during the ITI, the $20+5$ - discrimination would be acquired more readily than $5+$ $20-$.

\section{Method}

Subjects, apparatus, and procedure The subjects were 16 experimentally naïve male rats that were housed in the same manner as in Experiment 1. Their mean free-feeding weight was $256 \mathrm{~g}$; they were gradually reduced to between $80 \%$ and $85 \%$ of their free-feeding weights prior to training and were maintained at these weights by being fed a restricted diet after each experimental session. The experiment was conducted in the same conditioning chambers described in Experiment 1.

In the first two sessions, rats were trained to approach the food well in order to obtain sucrose solution in the manner described for Experiment 1, except that the screen was filled
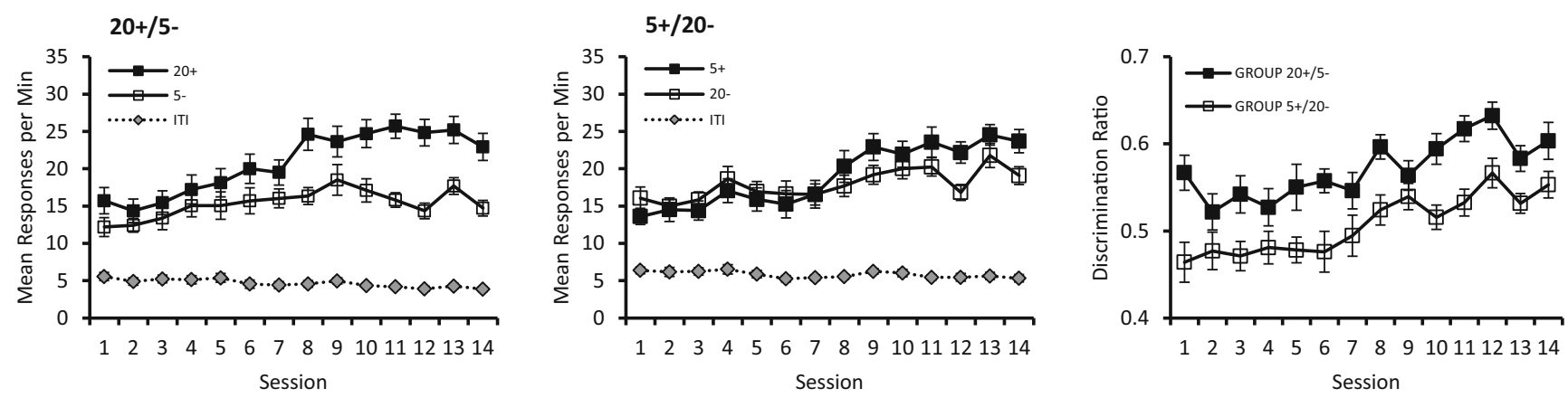

Fig. 2 Mean rates of responding to CS + and CS- for the 14 sessions of training for the $20+/ 5-$ (left-hand panel) and 5+/20- (center panel) groups of Experiment 1. The right-hand panel shows the discrimination ratios derived from the mean rates of responding. Error bars represent \pm 1 SEM. 
with alternating black and white horizontal bars that were each $4 \mathrm{~cm}$ high. The modification was made with the intention of ensuring that any associations formed during magazine training would involve black and white stimuli equally. These associations, if they formed, were then expected to exert a similar influence on responding during the various patterns introduced at the outset of discrimination training.

After magazine training, the rats were given 14 sessions of discrimination training with the same patterns containing five and 20 squares as in Experiment 1. Sucrose was presented after the patterns with the 20 squares, but not after five squares, for the $20+/ 5$ - group, whereas for the 5+/20- group sucrose was presented after displays with five, but not with 20 , squares on the screen. Throughout every ITI, the white screen displayed 80 black squares scattered throughout a notional rectangle $28 \mathrm{~cm}$ wide and $22 \mathrm{~cm}$ high located in the center of the screen. The squares, which were identical to those in the training stimuli, were separated by a mean distance of $1.8 \mathrm{~cm}(S D=0.8 \mathrm{~cm})$. The same pattern of 80 squares was used for every ITI. The remaining details concerning the training protocol, and the recording of the results, were the same as for Experiment 1.

\section{Results}

The mean rates of responding to $\mathrm{CS}+$ and $\mathrm{CS}-$ over the 14 sessions of discrimination training can be seen for the two groups in the left-hand and center panels of Fig. 3. It is apparent that neither group found the discrimination easy to solve, but toward the end of training the rate of responding was faster during CS + than during CS- for the 5+/20- group, whereas a similar difference failed to emerge in the 20+/5- group, even by the end of training. The discrimination ratios shown in the right-hand panel of the figure support these observations. The group mean ratios for the $20+/ 5-$ group failed to rise consistently above .50 throughout the experiment, whereas those for the 5+/20 - group increased gradually above this value as training progressed. An ANOVA conducted on the individual mean discrimination ratios for each of the 14 sessions confirmed the significant main effects of group, $F(1,14)=7.66, p$
$=.015, \eta_{\mathrm{p}}{ }^{2}=.35,95 \% \mathrm{CI}[.01, .60]$, and of session, $F(13,182)$ $=3.43, p<.001, \eta_{\mathrm{p}}{ }^{2}=.20,95 \%$ CI $[.05, .24]$, but the Session $\times$ Group interaction was not significant, $F<1$.

The left-hand and center panels of Fig. 3 also show that the mean rates of responding during the ITI were low for both groups. An ANOVA revealed no significant difference between the groups, $F<1$. The main effect of session, $F(13$, $182)=5.30, p<.001, \eta_{\mathrm{p}}{ }^{2}=.27,95 \%$ CI $[.12, .33]$, and the Session $\times$ Group interaction, $F(13,182)=2.51, p=.003, \eta_{\mathrm{p}}{ }^{2}=$ $.15,95 \%$ CI $[.02, .19]$, were found to be significant. Further analysis of the significant interaction revealed a significant effect of session for the $5+/ 20$ - group, $F(13,182)=6.31, p$ $<.001$, but not for the $20+/ 5-$ group, $F(13,182)=1.50, p>$ .10. In addition, there was a significant difference between the groups on Sessions 1 and $2, F_{\mathrm{s}}(1,196)>5.98$.

\section{Discussion}

As we predicted from the proposals of Kosaki et al. (2013), the presence of numerous black squares on the white screen of the monitor during the ITI resulted in the discrimination between five and 20 black squares being easier when the delivery of the reinforcer was signaled by five squares in the $5+/ 20$ - group rather than by 20 squares in the $20+/ 5-$ group. Indeed, we found no hint that the $20+/ 5-$ group was able to solve the discrimination.

When considering the poor performance of the $20+/ 5-$ group, it is worth noting that throughout the experiment responding in this group was considerably lower during the ITI than during the reinforced training trials with 20 squares. It is thus clear that the failure of this group to solve the discrimination was not due to an inability to distinguish between the array of squares presented during the ITI and the array presented during the reinforced training trials. Apart from the possibility that insufficient training was given in order to allow enough time for the $20+/ 5$ - group to solve the discrimination, it is hard to offer a satisfactory explanation for the poor performance of this group. As we noted earlier, the design of the present experiment was very similar to one conducted with
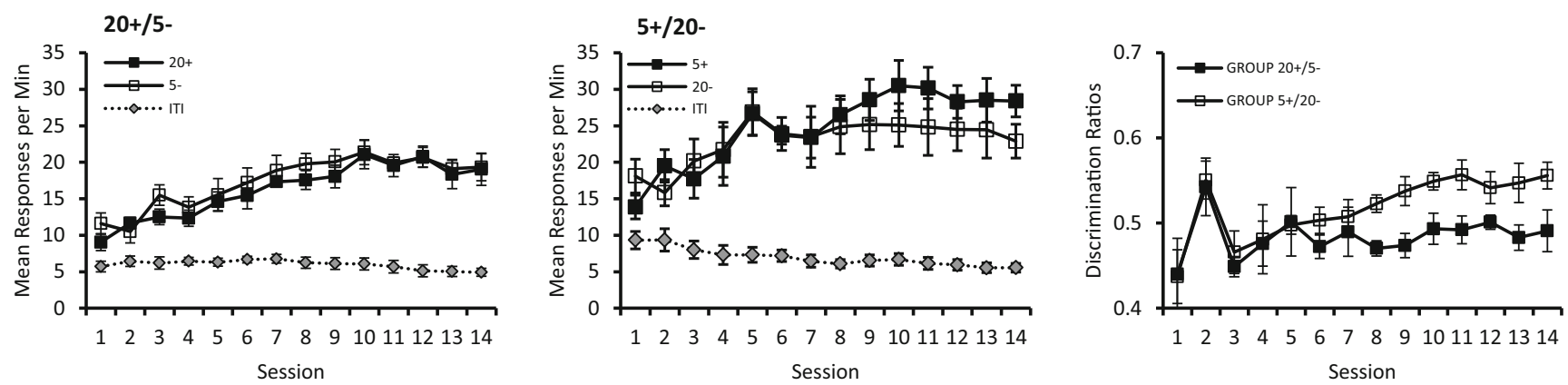

Fig. 3 Mean rates of responding to CS + and CS- for the 14 sessions of training for the $20+/ 5-$ (left-hand panel) and 5+/20- (center panel) groups of Experiment 2. The right-hand panel shows the discrimination ratios derived from the mean rates of responding. Error bars represent \pm 1 SEM. 
pigeons by Inman et al. (2015). In keeping with the present results, they found that the group trained with five squares as the CS+ solved the discrimination, albeit slowly, but the group trained with 20 squares as the CS+ failed completely to solve the discrimination. It is always dangerous to place too much emphasis on a null result, but according to the proposals of Kosaki et al. there is no good reason why the $20+/ 5-$ group in the present study, and the equivalent group in the study by Inman et al., should have failed to solve its discrimination. The results from the two experiments, therefore, may be regarded as posing a potential challenge to this account for the asymmetry that is seen with discriminations based on stimulus magnitude. Even though it may not be possible to provide a wholly satisfactory account of the present results, their close similarity with those reported by Inman et al. confirms their reliability and generality.

\section{Experiment 3}

The asymmetry in the discrimination of quantity that was observed in the first two experiments has been attributed to differences between the similarity of the ITI cues and the stimuli that serve as the $\mathrm{CS}+\mathrm{s}$ in the two discriminations (Kosaki et al., 2013). If this proposal is correct, then it should be possible to prevent the asymmetry from developing by presenting the CS+ and CS- of the discrimination without separating them by an ITI. Experiment 3 was conducted with this rationale in mind.

The most direct way of testing the foregoing prediction would be to repeat Experiment 1, say, with the ITI removed, and therefore with alternating 15 -s presentations of CS+ and CS-. Preliminary investigation revealed that this method of training did not result in the successful acquisition of the discrimination. After further investigation, the following technique was adopted (see Fig. 4). The two groups of rats that were trained without an ITI received alternating exposure to patterns with either five or 40 black squares throughout each experimental session. Sucrose was signaled by patterns with 40 squares, but not by five, in the $40+/ 5-/$ no-ITI group, and by patterns with five squares, but not by 40 , in the $5+/ 40-/$ no-ITI group. The duration of CS+ was always $73 \mathrm{~s}$, and the duration of CS- was three times this length. Sucrose solution was presented at irregular intervals on three occasions during each $\mathrm{CS}+$ trial. This training was intended to result in responding during $\mathrm{CS}+$ to be more frequent than during CS-. Moreover, on the basis of the proposals of Kosaki et al. (2013), the acquisition of this discrimination was expected to progress at the same rates in both groups.

Two additional groups were included in the experiment. The duration of each trial with CS+ was the same as for the groups just described, but the duration of CS- was reduced to $73 \mathrm{~s}$, so that it was the same as for CS+. Each of these trials was separated by an ITI of $73 \mathrm{~s}$, during which the screen on the monitor was entirely white. For the 40+/5-/ITI group, sucrose was presented during trials with the 40 -square patterns, but not with the five-square patterns, whereas for the 5+/40-/ITI group, sucrose was presented during patterns with five, but not with 40 , squares. The presence of the white screen during the ITI was expected to influence the two discriminations in the manner predicted by Kosaki et al. (2013), with the result that the discrimination would be acquired more rapidly by the 40+/5-/ITI than by the 5+/40-/ITI group.

\section{Method}

Subjects and apparatus For the experiment, we used 64 experimentally naïve male rats housed in the same manner as in the previous experiments. They were reduced to between $80 \%$ and $85 \%$ of their free-feeding weights $(M=234 \mathrm{~g})$ and were maintained at this level by being fed a restricted diet after each experimental session. The experiment was conducted in the chambers used in Experiments 1.

Procedure Rats were first given two sessions in which they were trained to retrieve sucrose when it was delivered into the food well. The full procedural details are as described in Experiment 1. For the duration of each of these sessions, the computer monitor was turned off. The rats were subsequently given ten further sessions in which they were trained to discriminate between the 40 - and five-square stimuli. The fivesquare stimuli were the same as those for Experiments 1 and 2. The 40-square stimuli consisted of 40 black squares, with sides of $1 \mathrm{~cm}$. The mean distance between the squares was $2.1 \mathrm{~cm}(S D=0.6)$. Each session comprised nine presentations of CS+ and nine presentations of CS- in an alternating fashion. The stimulus type presented first was determined at random, but was kept the same across groups for each session.

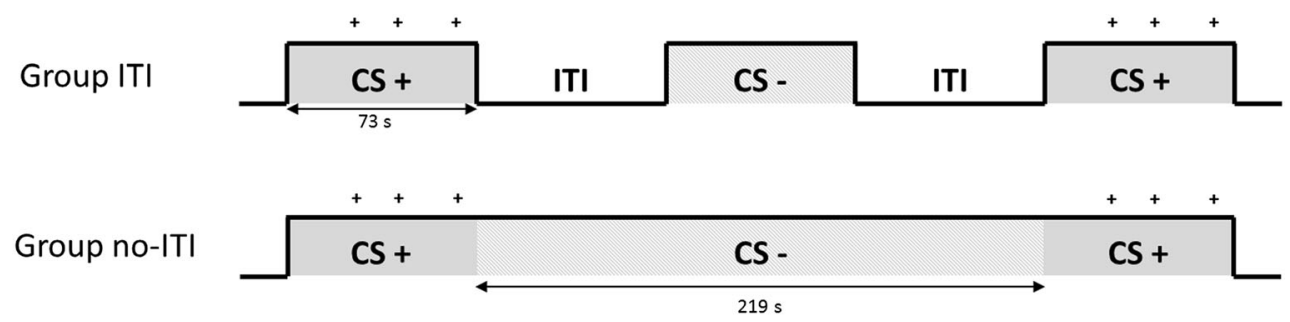

Fig. 4 Design of Experiment 3. The + symbols represent deliveries of $1 \mathrm{ml}$ of sucrose. 
For all groups, during the presentation of each CS+ sucrose was delivered at a randomly determined time between 1 and $20 \mathrm{~s}$ within each of three successive 20 -s periods. The first of these 20 -s periods occurred after a 10 -s interval during which no sucrose was delivered. Each 20 -s period was separated by $1 \mathrm{~s}$ in which no sucrose was delivered. Thus, each CS + presentation lasted $73 \mathrm{~s}$. For the two groups trained with an ITI, the duration of each trial with CS- was $73 \mathrm{~s}$, and each trial was separated by an interval of $73 \mathrm{~s}$ when the screen was entirely white. For the groups trained without an ITI, the duration of each trial with CS- was 219 s, and no interval was interposed between successive trials.

The number of snout entries prior to the first delivery of sucrose (pre-US) was recorded for every CS + trial. The timer that was in operation during $\mathrm{CS}+$, to determine when sucrose was first presented on each trial, was also in operation during CS-, but its purpose was solely to identify the end of the interval during which responding was recorded for the nonreinforced trials. Responses were also recorded throughout every ITI for the two groups trained with an ITI.

\section{Results}

Figure 5 presents the mean rates of pre-US responding to $\mathrm{CS}+$ and $\mathrm{CS}-$ across the ten sessions of training for the four groups.
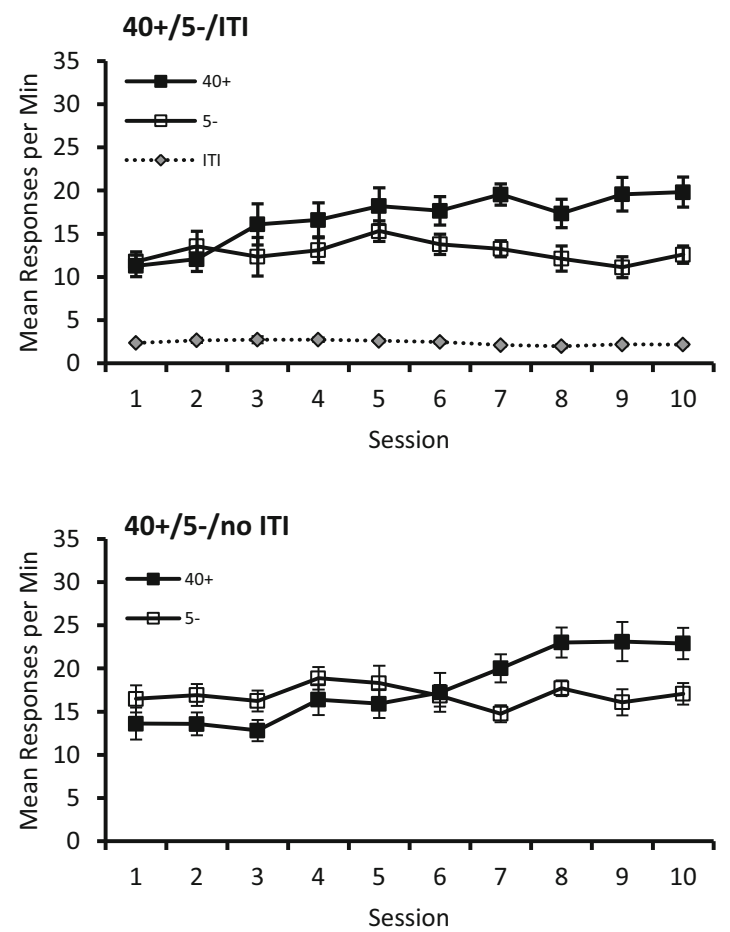

Fig. 5 Mean rates of responding to $\mathrm{CS}+$ and $\mathrm{CS}-$ for the ten sessions of training for the $40+/ 5-$ (left-hand panels) and 5+/40- (right-hand panels) groups of Experiment 3. The top panels present responding for the ITI
The discrimination was solved successfully by each group. The results displayed in the upper two panels of the figure are for the groups trained with an ITI, and it is evident that the group that received the $40+/ 5-$ discrimination mastered its problem more successfully than the group receiving the $5+/$ 40- discrimination. In keeping with these observations, the group mean discrimination ratios, shown in the left-hand panel of Fig. 6, were larger for the 40+/5-/ITI group than for the $5+/ 40-/$ ITI group for all but one session of training.

Turning now to the lower two panels of Fig. 5, which display the results from the two groups trained without an ITI, the courses of acquisition of the two discriminations are remarkably similar. Given this pattern of results, it should not be surprising to see in the right-hand panel of Fig. 6 that throughout the experiment there was not a substantial difference between the discrimination ratios for the $40+/ 5-/$ no-ITI group and the 5+/40-/no-ITI group.

A three-way ANOVA of the individual mean discrimination ratios for each of the ten sessions, with the factors of ITI (present or absent), Group, and Session, revealed significant main effects of ITI, $F(1,60)=16.59, p<.001, \eta_{\mathrm{p}}{ }^{2}=.22,95 \%$ CI $[.06, .38]$, and group, $F(1,60)=5.94, p=.018, \eta_{\mathrm{p}}{ }^{2}=.09$, $95 \%$ CI $[.00, .24]$, and importantly, a significant ITI $\times$ Group interaction, $F(1,60)=6.61, p=.013, \eta_{\mathrm{p}}{ }^{2}=.10,95 \%$ CI $[.00$, $.25]$, supporting the observation that there was an asymmetry in the acquisition of the discriminations for the groups trained with an ITI, but not for those trained without an ITI. Further
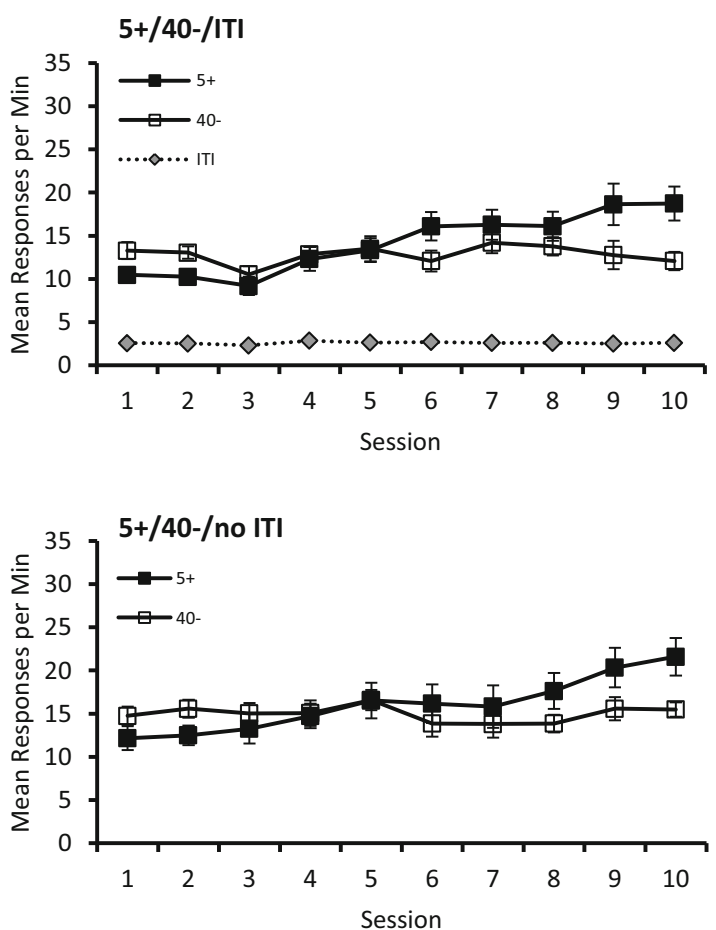

groups, and the lower panels present responding for the no-ITI groups. Error bars represent \pm 1 SEM. 

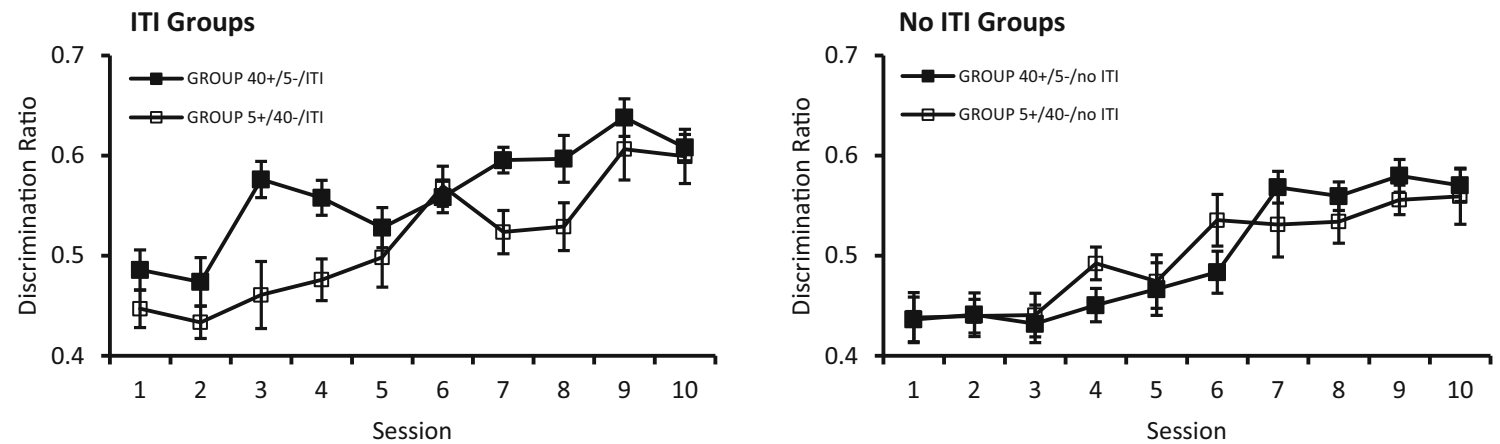

Fig. 6 Discrimination ratios for the ten sessions of training for the ITI (left-hand panel) and no-ITI (right-hand panel) groups in Experiment 3. Error bars represent $\pm 1 S E M$.

investigation of this significant interaction revealed a significant effect of group for rats trained with an ITI, $F(1$, $60)=21.89, p<.001, \eta_{\mathrm{p}}^{2}=.27,95 \%$ CI $[.09, .43]$, but not for the rats trained without an ITI, $F(1,60)=1.09, p$ $>$.10. We also observed a significant effect of ITI for the groups trained with 40 squares as $\mathrm{CS}+, F(1,60)=12.58$, $p=.001, \eta_{\mathrm{p}}{ }^{2}=17,95 \%$ CI $[.03, .34]$, but not for those trained with five squares as $\mathrm{CS}+, F<1$. The remaining results from the three-way ANOVA were a significant effect of session, $F(9,540)=26.76, p<.001, \eta_{\mathrm{p}}^{2}=.31$, $95 \%$ CI $[.24, .36]$, but the interactions of Session $\times$ ITI, $F(9,540)=1.18, p>.10$, Session $\times$ Group, $F(9,540)=$ $1.51, p>.10$, and Session $\times$ ITI $\times$ Group, $F(9,540)=$ $1.05, p>.10$, were not significant.

To explore further the lack of a significant difference between the ratios for Group 40+/5-/no-ITI and Group 5+/40-/no-ITI, a Bayesian analysis was conducted on the mean discrimination ratios across the ten sessions. This analysis could tell us whether the data favored the null hypothesis or the alternative hypothesis. A value above 3 has been suggested to be the cutoff for accepting whether the results substantially favor the null hypothesis, suggesting that the null hypothesis is three times more likely than the alternative hypothesis, given the data and priors (Rouder, Speckman, Sun, Morey, \& Iverson, 2009). The analysis revealed a Bayes Factor of 2.97 , which is just short of the conventional cutoff value and is thus suggestive of a pattern of results in favor of the null hypothesis.

Figure 5 also presents the rates of responding during the ITI for the ITI groups. An ANOVA revealed no difference in the rates of responding between the groups, $F<1$, although the effect of session, $F(9,270)=2.42, p=.012, \eta_{\mathrm{p}}{ }^{2}=.07,95 \%$ CI $[.00, .11]$, and the Session $\times$ Group interaction, $F(9,270)=$ $2.50, p=.009, \eta_{\mathrm{p}}{ }^{2}=.08,95 \%$ CI $[.01, .11]$, were significant. Further analysis of the significant interaction revealed an effect of session for the 40+/5- group, $F(9,270)=3.91, p<$ .001 , but not for the $5+/ 40-$ group, $F(9,270)=1.02, p=.42$. We also found a significant effect of group at Session $8, F(1$, $300)=4.08$.

\section{Discussion}

Despite the substantial differences between the treatment given to the two groups trained with an ITI in the present experiment and that given to the two groups of Experiment 1, the results from both pairs of groups were similar. In each experiment an asymmetry was observed, with a magnitude discrimination based on quantity being acquired more readily when sucrose was signaled by the larger rather than the smaller of the two stimuli. The new finding to emerge from the present experiment is that this asymmetry is less likely to occur when there is no interval between successive trials of the discrimination.

The design of the present experiment ensured that the four groups experienced the same amount of exposure to CS+, the same pattern of delivery of sucrose throughout the experimental session, and the same duration of each experimental session. As a consequence, there was a difference between the groups not only in whether they experienced an ITI, but also in the amount of exposure to the CS-. The groups trained without an ITI received three times as much exposure to CS- as the groups trained with an ITI. It is thus conceivable that the failure of the $40+/ 5-$-no-ITI group to acquire its discrimination more readily than the 5+/40-/no-ITI group was not due to the absence of the ITI, but to the excessive exposure to CS-. We are unable to rule out fully this explanation for our results, but the lack of any theoretical account of why excessive exposure to CS- should exert such an effect makes the explanation unlikely.

The failure to find an asymmetry in the discriminations of magnitude in the groups trained without an ITI stands in contrast to findings reported by Bouton and Hendrix (2011; see also Bouton \& García-Gutiérrez, 2006). Their rats received appetitive Pavlovian conditioning in which a single CS was preceded by either a short- or a long-duration ITI. For one group, food was presented after the CS when it was preceded by a long but not by a short ITI, and for a second group food was delivered when the CS was preceded by a short but not by a long ITI. The difference between the rates of responding on reinforced and nonreinforced trials during the CS was 
considerably greater when the delivery of food followed the longer rather than the shorter ITI. These results thus provide a further demonstration of the asymmetry observed in the acquisition of magnitude discriminations. However, because the cues that signaled whether food would be presented were present throughout the ITI, there was no period of exposure to context in the absence of the cues on which the discrimination was based. Contrary to our Experiment 3, therefore, this result demonstrates that an ITI is not essential for an asymmetry in the acquisition of a magnitude discrimination to be observed.

It is difficult to offer a satisfactory explanation for the discrepancy between the present results and those of Bouton and Hendrix (2011) and Bouton and García-Gutiérrez (2006), apart from suggesting that the asymmetry in discriminations based on stimulus magnitude is a result of more than one influence. Perhaps the principles responsible for the asymmetry found with magnitude discriminations based on quantity are different from those based on temporal duration. Why this should be the case remains to be determined.

\section{General discussion}

The experiments here have demonstrated for the first time with rats an asymmetry in the acquisition of a magnitude discrimination based on quantity. Experiments 1 and 3 revealed that a discrimination based on appetitive conditioning was relatively easy to acquire when the outcome was signaled by a large but not by a small number of identical objects, and relatively hard to acquire when the outcome was signaled by a small but not by a large number of objects. The principal purpose of the experiments was to determine whether this pattern of results occurs because the ease with which a magnitude discrimination is solved is related to the similarity between the $\mathrm{S}+$ and the stimuli present during the ITI. When both sets of cues are similar, then, according to Kosaki et al. (2013), the discrimination will be harder to solve than when they are different. Experiment 2 lent support to this possibility by showing that when the cues during the ITI were more similar to the CS composed of a large rather than a small number of objects, then the asymmetry was reversed. Experiment 3 further demonstrated the importance of the role played by the cues present during the ITI for the asymmetry in magnitude discriminations, by showing that the asymmetry was eliminated when training took place in the absence of an ITI.

Why should exposure to cues during the ITI be important for the asymmetry that was observed in the above magnitude discriminations? In order to answer this question, Inman et al. (2015) attempted to explain their findings with reference to two different theories of learning: the Rescorla and Wagner (1972) theory and the configural theory of Pearce (1987, 1994). To render the experiments amenable to analysis with these theories, they followed the lead of Bouton and Hendrix (2011) by treating a magnitude discrimination as one in which a low-magnitude stimulus is represented by one element, A, and a high-magnitude stimulus is represented by two elements, AB. Thus, a 5+/20- discrimination can be represented as $\mathrm{A}+/ \mathrm{AB}-$, and a $20+/ 5-$ discrimination as $\mathrm{AB}+/ \mathrm{A}-$. If we are to acknowledge the role of the cues present during the ITI, $\mathrm{C}$, in the solution of these discriminations, then, because these cues were also present during the training trials, a $5+/ 20$ discrimination becomes $\mathrm{C}-/ \mathrm{CA}+/ \mathrm{CAB}-$, and $20+/ 5$ - becomes $\mathrm{C}-/ \mathrm{CAB}+/ \mathrm{CA}-$. Using this characterization, Inman et al. reported the results of computer simulations showing that it is possible to predict the results from Experiment 1 using Pearce's (1994) theory. During a discrimination, according to this theory, the different patterns of stimulation, including that provided by the ITI, enter into associations with the outcome with which they are paired. Thus, a discrimination of the kind $\mathrm{C}-/ \mathrm{CA}+/ \mathrm{CAB}-$ will result in $\mathrm{CA}$ entering into an excitatory association that will generalize to $\mathrm{C}$ because of their similarity. The nonreinforced ITI stimulation, $\mathrm{C}$, will then enter into an inhibitory association in order to counter the excitation that generalizes to it. This inhibition will, in turn, generalize to $\mathrm{CA}$, and by virtue of disrupting the manifestation of its excitatory properties, hinder the acquisition of the $\mathrm{A}+/ \mathrm{AB}-$ discrimination. A similar process will occur in the group trained with $\mathrm{C}-/ \mathrm{CAB}+/ \mathrm{CA}-$. The considerable difference between $\mathrm{C}$ and $\mathrm{CAB}$ will, however, result in relatively little generalization of the excitation from $\mathrm{CAB}$ to $\mathrm{C}$, which will then need to acquire rather little inhibition. Moreover, little of this inhibition will then generalize to $\mathrm{CAB}$, and its disruptive impact on the $\mathrm{AB}+/ \mathrm{A}-$ discrimination will be minimal. As a consequence, the theory predicts that the $\mathrm{AB}+/ \mathrm{A}$ - discrimination will be acquired more readily than $\mathrm{A}+/ \mathrm{AB}-$.

Turning to Experiment 2, the characterization of the training treatment would be the same as that just described, except that the stimulation during the ITI would be CABD-, to take account of the larger number of squares than during either training stimulus. Once again, computer simulations reported by Inman et al. (2015) revealed that the theory of Pearce (1994) can predict the asymmetry that was observed in the experiment. When it comes to Experiment 3, the removal of the ITI would result in the training being characterized as $\mathrm{CAB}+/ \mathrm{CA}-$ and $\mathrm{CA}+/ \mathrm{CAB}-$. In these circumstances, the theory predicts that both discriminations would be acquired at the same rate.

The foregoing analysis has two implications for the rate of responding during the ITI. First, as the inhibition that is predicted to be associated with the cues present during the ITI gains in strength, so responding during these intervals would become progressively weaker. Second, the rate of responding during the ITI is predicted to be stronger for the groups trained with the ITI cues more similar to $\mathrm{S}+$ than to $\mathrm{S}-$-, than for the groups trained with the opposite arrangement. Unfortunately, the overall pattern of results from the three experiments makes 
it hard to evaluate these predictions. Thus, both predictions were confirmed in Experiment 1, but the small within-group and between-group differences do not present compelling support for the theory from which the predictions were derived. In Experiment 2, the between-group differences were opposite to those predicted, in that only one group showed a clear decline in responding as training progressed. Finally, in Experiment 3, the ITI response rates of approximately two responses per minute were so slow that it is debatable whether any meaningful theoretical conclusions can be drawn from them.

In order to explore the predictions made by the Rescorla and Wagner (1972) theory concerning the role of ITI cues in the asymmetry of magnitude discriminations, Inman et al. (2015), conducted a series of computer simulations using the same characterization of the experimental designs as for the simulations with the theory of Pearce (1994). The simulations revealed that the theory was able to predict the asymmetries found in Experiments 1 and 2, but, as far as Experiment 3 is concerned, it predicts that an asymmetry should have been observed with the no-ITI groups as well. The removal of the ITI means that the training can be characterized as $\mathrm{CAB}+$ / $\mathrm{CA}-$ or $\mathrm{CA}+/ \mathrm{CAB}-$. The first of these discriminations is essentially a feature-positive discrimination, and the second is a feature-negative discrimination. As Bouton and Hendrix (2011) pointed out, the Rescorla-Wagner theory predicts the former will be acquired more readily than the latter.

A prediction that is common to both of the theories that have been considered is that the $20+5$ - discrimination in Experiment 2 would be solved, despite the presence of a large number of squares shown on the screen during the ITI. Inspection of the left-hand panel of Fig. 3 reveals that this prediction was not confirmed, since there was no hint of the discrimination being solved, even after 14 sessions of training. Inman et al. (2015) described a similar failure to solve the equivalent discrimination with pigeons. It is not clear whether this failure to solve the discrimination was simply a consequence of the use of an insufficiently sensitive methodology, or whether it points to a weakness in our theoretical understanding of how magnitude discriminations are solved.

The experiments have shown for the first time that rats can discriminate between different quantities of the same visual stimulus, a black square, which raises the question of how the discrimination was solved. One possibility is that the discrimination was solved by referring to the number of squares that were displayed on the screen. A second possibility is that the discrimination was based on the distance between the squares, which was greater for the five-square than for the 20-square patterns. Finally, there is the possibility that the discrimination was based on the amount of black, or the amount of white, stimulation provided by the patterns: The 20 -square patterns contained more black, and less white, than the five-square patterns. On the basis of the present evidence, it is not possible to choose between these alternatives (although some research has stated that rats preferentially discriminate on the basis of luminance; e.g., Minini \& Jeffery, 2006), but that does not detract from the principal conclusion that we wish to draw from the experiments. The stimulation that is present during the ITI plays a role in the asymmetry that is found with discriminations based on stimulus magnitude, but a full theoretical understanding of how this role is effective remains to be developed.

Author note This research was supported in part by a grant from the United Kingdom Biotechnology and Biological Sciences Research Council (Grant No. BB/H006176).

Open Access This article is distributed under the terms of the Creative Commons Attribution 4.0 International License (http:// creativecommons.org/licenses/by/4.0/), which permits unrestricted use, distribution, and reproduction in any medium, provided you give appropriate credit to the original author(s) and the source, provide a link to the Creative Commons license, and indicate if changes were made.

\section{References}

Bouton, M. E., \& García-Gutiérrez, A. (2006). Intertrial interval as a contextual stimulus. Behavioural Processes, 71, 307-317.

Bouton, M. E., \& Hendrix, M. C. (2011). Intertrial interval as a contextual stimulus: further analysis of a novel asymmetry in temporal discrimination learning. Journal of Experimental Psychology: Animal Behavior Processes, 37, 79-93.

Inman, R. A., Honey, R. C., \& Pearce, J. M. (2015). Asymmetry in the discrimination of quantity: the role of stimulus generalization. Journal of Experimental Psychology: Animal Learning and Cognition. doi:10.1037/xan0000073

Kosaki, Y., Jones, P. M., \& Pearce, J. M. (2013). Asymmetry in the discrimination of length during spatial learning. Journal of Experimental Psychology: Animal Behavior Processes, 39, 342 356. doi: $10.1037 / \mathrm{a} 0032570$

Kyd, R. J., Pearce, J. M., Haselgrove, M., Amin, E., \& Aggleton, J. P. (2008). The effects of hippocampal system lesions on a novel temporal discrimination task for rats. Behavioural Brain Research, 187, 159-171. doi:10.1016/j.bbr.2007.09.010

Minini, L., \& Jeffery, K. J. (2006). Do rats use shape to solve "shape discriminations"? Learning and Memory, 13, 287-297. doi:10. 1101/lm.84406

Pearce, J. M. (1987). A model of stimulus generalization for Pavlovian conditioning. Psychological Review, 94, 61-73. doi:10.1037/0033295X.94.1.61

Pearce, J. M. (1994). Similarity and discrimination: A selective review and a connectionist model. Psychological Review, 101, 587-607. doi:10.1037/0033-295X.101.4.587

Pelz, C., Gerber, B., \& Menzel, R. (1997). Odorant intensity as a determinant for olfactory conditioning in honeybees: Roles in discrimination, overshadowing and memory consolidation. Journal of Experimental Biology, 200, 837-847.

Rescorla, R. A., \& Wagner, A. R. (1972). A theory of Pavlovian conditioning: Variations in the effectiveness of reinforcement and nonreinforcement. In A. H. Black \& W. F. Prokasy (Eds.), Classical conditioning II (pp. 64-99). New York, NY: Appleton-CenturyCrofts.

Rouder, J. N., Speckman, P. L., Sun, D., Morey, R. D., \& Iverson, G. (2009). Bayesian $t$ tests for accepting and rejecting the null 
hypothesis. Psychonomic Bulletin \& Review, 16, 225-237. doi:10. 3758/PBR.16.2.225

Steiger, J. H. (2004). Beyond the $F$ test: Effect size confidence intervals and tests of close fit in the analysis of variance and contrast analysis. Psychological Methods, 9, 164-182. doi:10.1037/1082-989X.9.2.164

Todd, T. P., Winterbauer, N. E., \& Bouton, M. E. (2010). Interstimulus interval as a discriminative stimulus: Evidence of the generality of a novel asymmetry in temporal discrimination learning. Behavioural Processes, 84, 412-420. doi:10.1016/j.beproc.2010.01.002
Vonk, J., \& Beran, M. J. (2012). Bears "count" too: Quantity estimation and comparison in black bears (ursus americanus). Animal Behaviour, 84, 231-238.

Watanabe, S. (1998). Discrimination of "four" and "two" by pigeons. Psychological Record, 48, 383-391.

Zieliński, K., \& Jakubowska, E. (1977). Auditory intensity generalization after CER differentiation training. Acta Neurobiologiae Experimentalis, 37, 191-205. 